\title{
Piezoresistance and magnetoresistance of composites based on manganite $\mathrm{La}_{0.7} \mathrm{Sr}_{0.3} \mathrm{MnO}_{3}$
}

\author{
Y.V. Kabirov ${ }^{\dagger, 1}$, A.S. Bogatin ${ }^{1}$, E. N. Sidorenko ${ }^{1}$, M. V. Belokobylsky ${ }^{1}$, A. S. Mikheykin ${ }^{1}$,
} A. O. Letovaltsev ${ }^{1}$, A. L. Bulanova ${ }^{1}$, N. V. Prutsakova ${ }^{2}$

†salv62@mail.ru

${ }^{1}$ Southern Federal University, 105/42 Bolshaya Sadovaya St., Rostov-on-Don, 344090, Russia

${ }^{2}$ Don State Technical University, 1 Gagarin Sq., Rostov-on-Don, 344010, Russia

\begin{abstract}
The development of new sensitive multifunctional materials, which simultaneously react both to mechanical pressure and a constant magnetic field, is one of challenges of the modern materials science. In order to create materials that can simultaneously possess extrinsic piezoresistance and magnetoresistance, polymer composites based on $\mathrm{La}_{0.7} \mathrm{Sr}_{0.3} \mathrm{MnO}_{3}$ (LSMO) and low density polyethylene (LDPE) of composition (100- $x$ )LSMO/ $x \mathrm{LDPE}$ at $x=7,8,9,12,15,17,18,22$ mass percent have been synthesized in this work. The composites have been synthesized at $170^{\circ} \mathrm{C}$ and a pressure of $50 \mathrm{MPa}$ in the presence of hydrocarbons. The phase composition of the obtained samples has been studied by X-ray diffraction and infrared spectroscopy. Electric, piezoresistance and magnetoresistance properties of the samples have been studied. It has been found out that with increasing concentration of polyethylene, the electrical resistance of the samples also increases. It has been established that the percolation threshold of the composites is about $13 \mathrm{wt} . \%$ of polyethylene. A maximum is observed in the dependence of the electrical resistance of the samples on the uniaxial pressure at a concentration of polyethylene near the percolation threshold at $x=15 \mathrm{wt} . \%$. Samples with concentration of $x=15 \mathrm{wt}$.\% of low density polyethylene show extrinsic piezoresistance of about $17 \%$ in the pressure range $0-270 \mathrm{kPa}$. Significant hysteresis is observed when considering the dependence of piezoresistance on pressure for all samples. The pressure sensitivity for a sample of $85 \% \mathrm{LSMO} / 15 \% \mathrm{LDPE}$ reaches $8.40 \mathrm{~m} \Omega / \mathrm{Pa}$. The maximum values of the negative isotropic tunnel magnetoresistance of the composites $88 \% \mathrm{LSMO} / 12 \% \mathrm{LDPE}$ have values of about $2 \%$ in a $15 \mathrm{kOe}$ magnetic field. We believe that the materials studied in the paper could be useful for designing multifunctional biosensors of mechanical pressure and constant magnetic field.
\end{abstract}

Keywords: manganite, composite, piezoresistance, magnetoresistance.

УДК: 537.9

\section{Пьезорезистивность и магниторезистивность полимерных композитов на основе манганита $\mathrm{La}_{0.7} \mathrm{Sr}_{0.3} \mathrm{MnO}_{3}$}

Кабиров Ю. В. ${ }^{\dagger, 1}$, Богатин А. С. ${ }^{1}$, Сидоренко Е. Н. ${ }^{1}$, Белокобыльский М. В. ${ }^{1}$,

\author{
Михейкин А.С. ${ }^{1}$, Летовальцев А. О. ${ }^{1}$, Буланова А. Л. ${ }^{1}$, Пруцакова Н. В. ${ }^{2}$ \\ ${ }^{1}$ Южный федеральный университет, ул. Большая Садовая, 105/42, Ростов-на-Дону, 344006, Россия \\ ${ }^{2}$ Донской государственный технический университет, пл. Гагарина, 1, Ростов-на-Дону, 344000, Россия
}

Одной из проблем современного материаловедения является создание новых чувствительных многофункциональных материалов, реагирующих одновременно как на давление, так и на постоянное магнитное поле. В нашей работе с целью создания материалов, обладающих несобственной пьезорезистивностью и магниторезистивностью одновременно, синтезированы полимерные композиты на основе манганита $\mathrm{La}_{0.7} \mathrm{Sr}_{0.3} \mathrm{MnO}_{3}$ (LSMO) и полиэтилена низкой плотности (LDPE) состава (100-x)LSMO/ $x$ LDPE при $x=7,8,9,12,15,17,18,22$ масс.\%. Синтез композитов проведен при температуре $170^{\circ} \mathrm{C}$ и давлении 50 МПа в присутствии углеводородов. Фазовый состав полученных образцов изучен методами рентгеновской дифракции и инфракрасной спектроскопии. Исследованы электрические, пьезорезистивные и магниторезистивные свойства образцов. Обнаружено, что при увеличении концентрации 
полиэтилена возрастает электрическое сопротивление образцов. Порог перколяции композитов определен около 13 масс.\% полиэтилена. Установлено, что в зависимости электрического сопротивления образцов от одноосного давления при концентрации полиэтилена вблизи порога перколяции при $x=15 \%$ наблюдается максимум. Образцы с концентрацией $x=15 \%$ проявляют несобственную пьезорезистивность порядка $17 \%$ в области давлений 0 - 270 кПа. На зависимости пьезорезистивности от давления для всех образцов наблюдается существенный гистерезис. Чувствительность к давлению для образца 85\%LSMO/15\%LDPE достигает 8.40 м $\Omega /$ Па. Максимальные значения отрицательной изотропной туннельной магниторезистивности композитов 88\%LSMO/12\%LDPE имеют величины порядка 2\% в магнитном поле 15 кЭ. Считаем, что на основе предложенных материалов могут быть созданы многофункциональные бисенсоры механического давления и постоянного магнитного поля.

Ключевые слова: манганит, композит, пьезорезистивность, магниторезистивность.

Допированный манганит $\mathrm{La}_{0.7} \mathrm{Sr}_{0.3} \mathrm{MnO}_{3} \quad$ (LSMO) известен как проводящее ферромагнитное соединение с высокой степенью спиновой поляризации носителей заряда и температурой Кюри 360 K [1]. На его основе синтезируются двухфазные композиты, обладающие при комнатной температуре магниторезистивными свойствами, связанными с межгранульным спинзависимым туннелированием электронов в постоянном магнитном поле [2]. Нужно отметить, что такие составы типа проводник/диэлектрик проявляют максимальные значения магниторезистивности вблизи порога перколяции [3]. В качестве второй, диэлектрической фазы могут быть использованы как неорганические материалы, так и органические [4 - 8]. Преимущества синтеза магниторезистивных материалов с полимерной матрицей очевидны - это исключение диффузионных процессов и химических реакций, характерных для традиционного твердофазного керамического синтеза во время приготовления композитов. Электрическое сопротивление подобных составов может значительно изменяться под воздействием внешнего давления в том случае, если упругие свойства компонентов композита существенно различаются. Например, эффект несобственной пьезорезистивности проявляется вблизи порога протекания при использовании в качестве диэлектрической упругой фазы полимера, a проводящей - графита, или малых частиц металлов $[5,6]$. Подобные сенсоры давления на основе несобственного пьезорезистивного эффекта не требуют усилителей и сложных преобразователей сигнала. Однако задача создания полимерных материалов на основе LSMO одновременно имеющих и пьезорезистивный, и магниторезистивный отклик не была поставлена и решена. В нашей работе впервые синтезированы и изучены пьезорезистивные, a также магниторезистивные свойства композитных материалов состава (100-x)LSMO/xLDPE при $x=7,8,9,12,15,17,18,22$ масс.\%, где LDPE low-density polyethylene, полиэтилен низкой плотности. Выбор полиэтиленовой матрицы обусловлен свойствами полиэтилена низкой плотности - высокой химической стабильностью, дешевизной, по сравнению с другими органическими соединениями, использованными в работах [4-9]. Манганит в виде поликристаллов LSMO был заранее синтезирован при температуре $1150^{\circ} \mathrm{C}$ и затем дополнительно измельчен. Следует отметить, что полиэтилен имеет упруго-эластичные свойства в некоторых пределах деформаций [10].

Тонкопленочный полиэтилен и порошок LSMO послойно (20-60 слоев) помещался в пресс-форму, где прессовался под давлением 1 ГПа. Далее образец в пресс-форме в присутствии бензина выдерживался для сваривания при температуре выше температуры плавления полиэтилена $165-170^{\circ} \mathrm{C}$ в течение 60 минут при небольшом давлении (50 МПа) вместе с электродами из фольги алюминия. После охлаждения до комнатной температуры образцы представляли собой диски диаметром 10 мм, и толщиной 2 мм. Массовые доли компонентов и оценка пористости образцов контролировались методом озоления при температуре $1000^{\circ} \mathrm{C}$ в воздушной атмосфере. Свойства образцов (плотность, пьезорезистивность), синтезированных по описанной технологии имеют удовлетворительную воспроизводимость. Для каждой концентрации из $x=7,8,9,12,15,17,18,22$ масс.\% полиэтилена синтезированы 5-7 образцов. Отклонения электрического сопротивления от средних значений серии не превышало 6-7\%.

Для характеризации образцов использована оптическая и электронная микроскопия (JEOL-JSM 6390LA), рентгеновская дифрактометрия (ДРОН-3М и ARL-X'TRA), ИК-спектроскопия (Фурье-инфракрасный спектрометр ФСМ-1202) в диапазоне 400 - $4000 \mathrm{~cm}^{-1}$, с разрешением $4 \mathrm{~cm}^{-1}$, усреднением по десяти спектрам.

Измерения электрического сопротивления серии образцов в зависимости от одноосного механического давления проведены двухэлектродным методом на калиброванной установке с точностью 0.5 кПа. Геометрия измерения - ток вдоль направления давления. Магниторезистивность была исследована по методике, описанной в [3]. Для определения типа проводимости исследованных образцов применялась методика, основанная на эффекте Зеебека.Кратковременное нагревание части образца, примыкающей к электроду, на который подается отрицательный потенциал от источника напряжения, вызывало возрастание тока в электрической цепи. Это свидетельствует об электронном типе проводимости всех исследованных образцов, а зависимость их проводимости от температуры имеет полупроводниковый характер.

На Рис. 1 представлена микрофотография одного из образцов.

Согласно электронно-микроскопическим исследованиям размеры кристаллитов LSMO находятся в диа- 


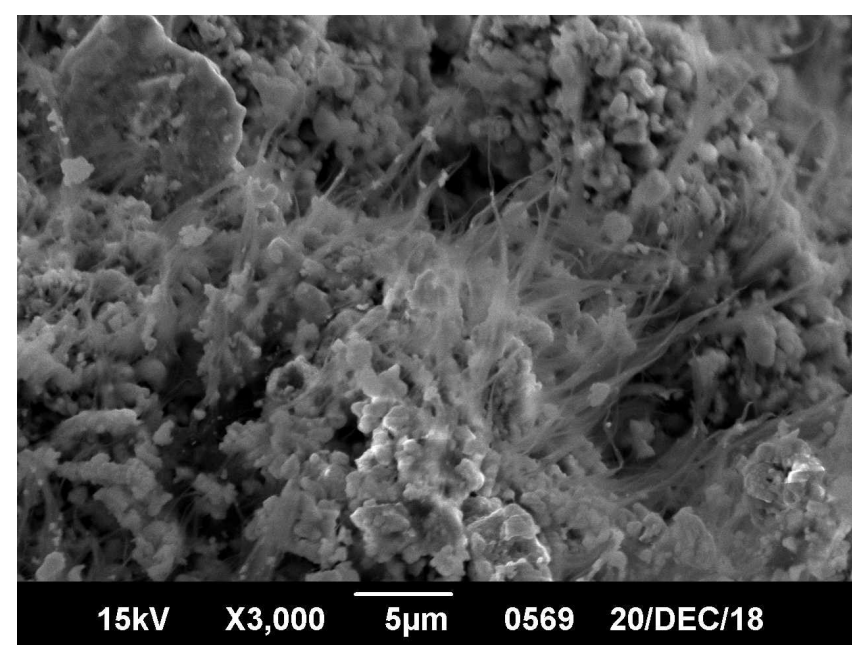

Рис.1.SEM-снимок поверхности скола образца 88\%LSMO/12\%LDPE. Fig. 1. SEM-image of the sample cleaved surface $88 \% \mathrm{LSMO} / 12 \% \mathrm{LDPE}$.

пазоне от 1 до 2 мкм. Объемная пористость $K$ образцов оценена согласно формуле:

$$
K=\left(\left(V_{R}-V_{C}\right) / V_{C}\right) \cdot 100 \%,
$$

где $V_{R}$ - объем реального образца, $V_{C}$ - расчетный объем образца согласно литературным данным плотности манганита $\left(6.43\right.$ г/ $\left.\mathrm{cm}^{3}\right)$ и полиэтилена $\left(0.92 \mathrm{r} / \mathrm{cm}^{3}\right)$. Массы манганита и полиэтилена получены из данных взвешивания манганита после озоления композитов. Объемная пористость $K$ для наших образцов имеет величину порядка $10 \%$.

На Рис. 2 приведена рентгенограмма одного из образцов, 88\%LSMO/12\%LDPE.

Данные рентгеновской дифракции свидетельствуют о наличии в синтезированных композитах $\mathrm{La}_{0.7} \mathrm{Sr}_{0.3} \mathrm{MnO}_{3}$ c пространственной группой симметрии $\mathrm{R} \overline{3} \mathrm{c}$ как основной кристаллической фазы с параметрами ячейки: $a=5.5054(5) \AA$ и $c=13.3598(17) \AA$. Профильный фактор недостоверности $R_{p}=2.68 \%$. Полученные значения параметров элементарной ячейки совпадают с известными литературными данными по структуре $\mathrm{La}_{0.7} \mathrm{Sr}_{0.3} \mathrm{MnO}_{3}$ с пространственной группой симметрии $\mathrm{R} \overline{3} \mathrm{c}$ и параметрами: $a=5.5086 \AA, c=13.362 \AA$ [11] до третьего и второго знака после запятой соответственно. На рентгенограммах образцов наблюдаются дифракционные отражения от примесей исходных реагентов - $\mathrm{La}_{2} \mathrm{O}_{3}(\mathrm{~L})$ и $\mathrm{Mn}_{2} \mathrm{O}_{3}(\mathrm{M})$ с содержанием 2-3 масс.\%, которые учитывались при анализе дифракционных данных ( $\mathrm{La}_{2} \mathrm{O}_{3}$ ICSD 100214 [12]; $\mathrm{Mn}_{2} \mathrm{O}_{3}$ ICSD 9090 [13]). Оценка средних значений размеров областей совершенства LSMO согласно соотношению Селякова-Шеррера дает величины около 120 нм. При этом дифракционных отражений от полиэтилена не наблюдается.

Инфракрасный спектр отражения образцов содержит линии, характерные для двух компонентов материала (Рис. 3).

Широкая линия в области частот $590 \mathrm{~cm}^{-1}$ характерна для манганитов с кристаллической структурой типа перовскита и соответствует одному из двух нормальных колебаний $\mathrm{MnO}_{6}^{3+}$ октаэдров, активных в ИК спектрах [14]. В спектре также наблюдаются моды, соответствующие полиэтилену [15]. Две линии в области с частотами 2851 и 2918 см $^{-1}$ соответствуют симметричным и антисимметричным валентным колебаниям $\mathrm{CH}_{2}$ групп. Деформационные (1464 и $1485 \mathrm{~cm}^{-1}$ ) и маятниковые колебания (720 и $731 \mathrm{~cm}^{-1}$ ) $\mathrm{CH}_{2}$ групп полиэтилена также заметны на спектре, но выражены существенно слабее.

Измерения показали, что электрическое сопротивление образцов увеличивается с ростом концентрации полиэтилена $(C)$. Порог перколяции композитов опре-

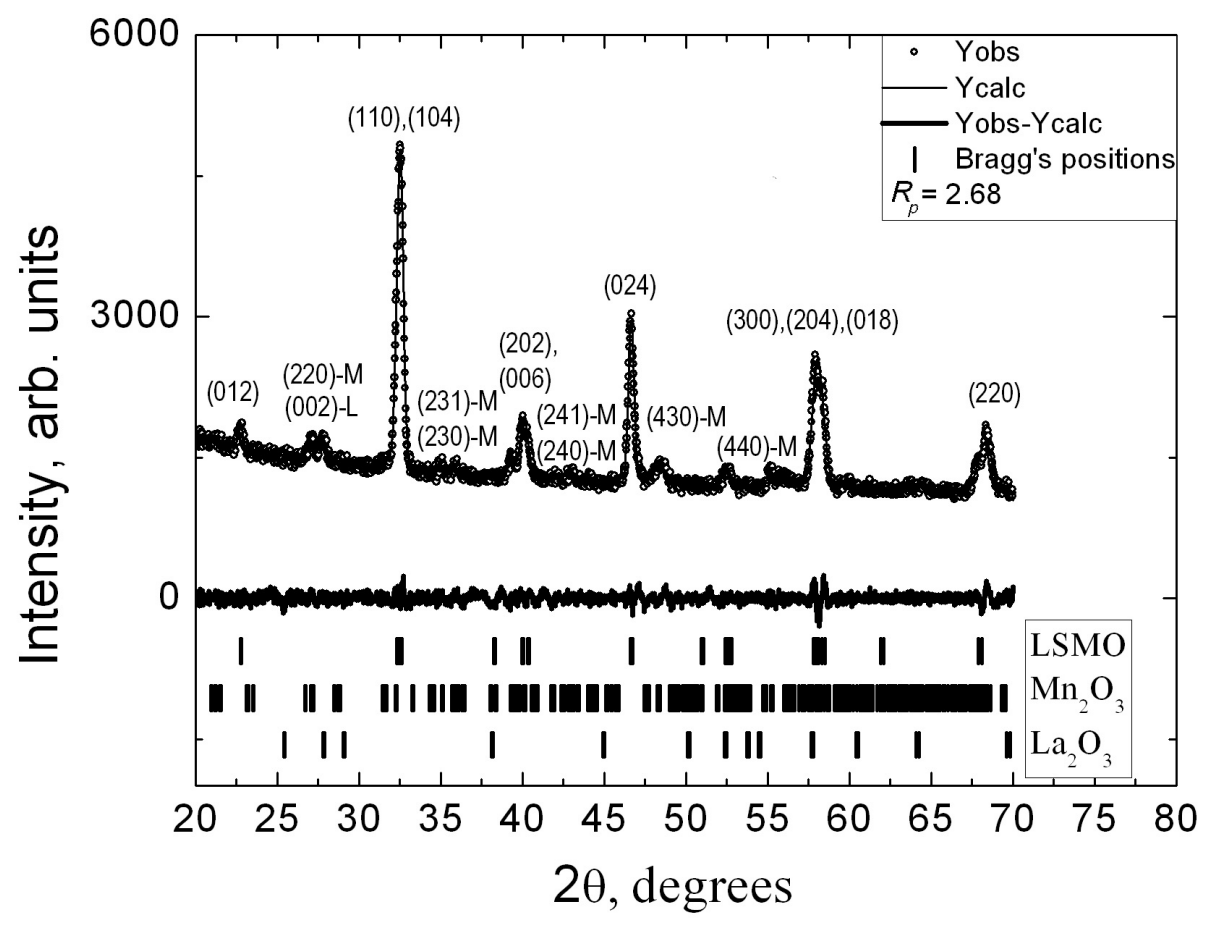

Рис. 2. Рентгенограмма образца $88 \% \mathrm{LSMO} / 12 \% \mathrm{LDPE}$.

Fig. 2. X-ray diffraction pattern of the sample $88 \%$ LSMO/12\%LDPE. 


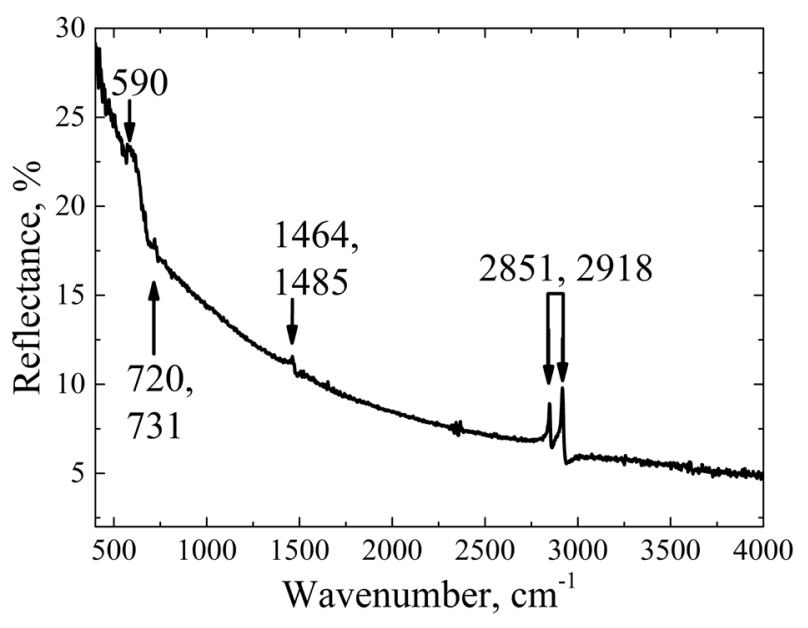

Pис. 3. ИК-спектр отражения образца 88\%LSMO/12\%LDPE. Fig. 3. Reflectance infrared spectrum of the sample $88 \%$ LSMO/12\%LDPE.

делен в области концентраций полиэтилена около $12-13$ масс.\%. Наибольший рост сопротивления образцов (в несколько раз - с 5-7 к $\Omega$ до 30 к $\Omega$ ) наблюдался в интервале $x=10-18$ масс.\%. Отметим, что порог протекания сильно зависит не только от массовых соотношений компонентов, но и от формы проводящих и диэлектрических частиц, и от технологии приготовления $[8,16]$. Для образцов с концентраций полиэтилена вблизипорогапротекания вольтамперныехарактеристики имеют параболический вид, Рис. 4 a. Вольтамперные характеристики остальных образцов близки к линейным, например, Рис. 4 b.

Для образца 88\%LSMO/12\%LDPE (Рис. 4 a) параболическая зависимость тока от приложенного напряжения, вероятно, отражает наличие туннельных барьеров между кристаллитами LSMO [17]. В исследованной области приложенного электрического напряжения остальные образцы имеют омические вольтамперные характеристики, (Рис. $4 \mathrm{~b})$.

Расчет пьезорезистивности $P R$ проводился по формуле:

$$
P R=\left(\left(R_{0}-R_{P}\right) / R_{0}\right) \cdot 100 \%,
$$

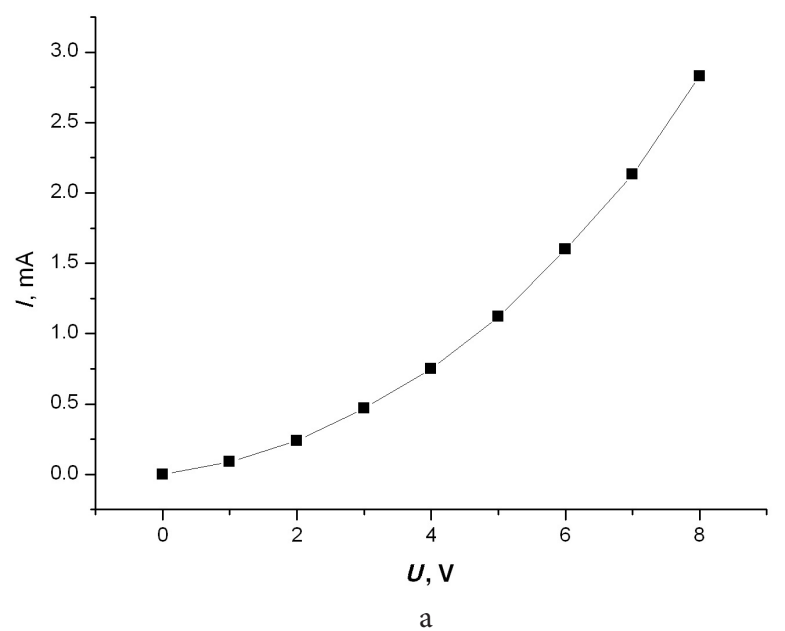

где $R_{0}$ - электрическое сопротивление образца без приложенного давления, $R_{p}-$ значение электрического сопротивления под давлением $P$. На Рис. 5 показана зависимость $P R$ от концентрации полиэтилена.

Следует отметить экстремальный характер зависимости пьезорезистивности приготовленных образцов от концентрации полиэтилена. Максимальное значение пьезорезистивности наблюдается вблизи порога протекания - для образца 85\%LSMO/15\%LDPE. На Рис. 6 представлена зависимость электрического сопротивления одного из образцов вблизи порога протекания от приложенного давления.

При одномерном сжатии исследуемого образца 85\%LSMO/15\%LDPE, находящегося вблизи концентрационного порога протекания, за счет деформации полиэтиленовой матрицы, в которой расположены кристаллиты манганита, уменьшается расстояние между кристаллитами. Поэтому сопротивление исследуемого образца также уменьшается.

Характер зависимости $R(P)$, показанный на Рис. 6, является гистерезисным. Однако после снятия давления значение электрического сопротивления восстанавливается. Время ожидания в каждой точке измерений

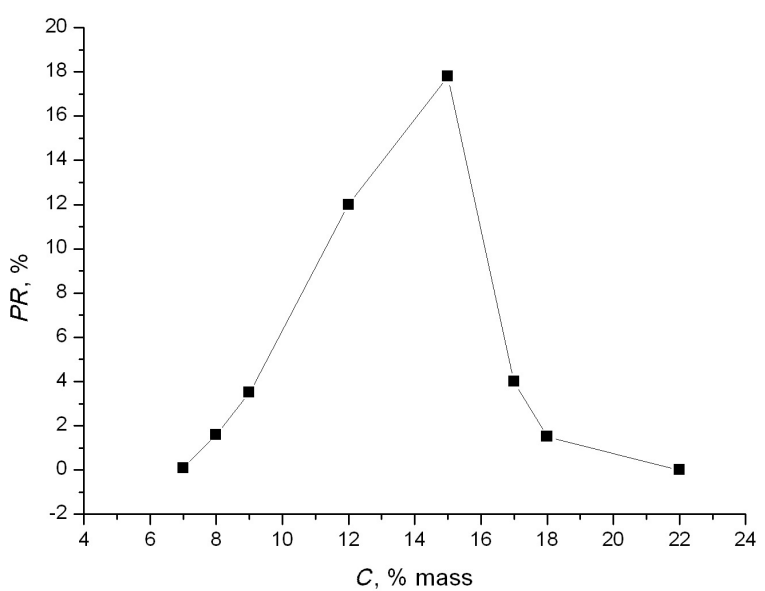

Рис. 5. Зависимость пьезорезистивности композитов от концентрации в них полиэтилена.

Fig. 5. The dependence of the piezoresistance of composites on the concentration of polyethylene in them..

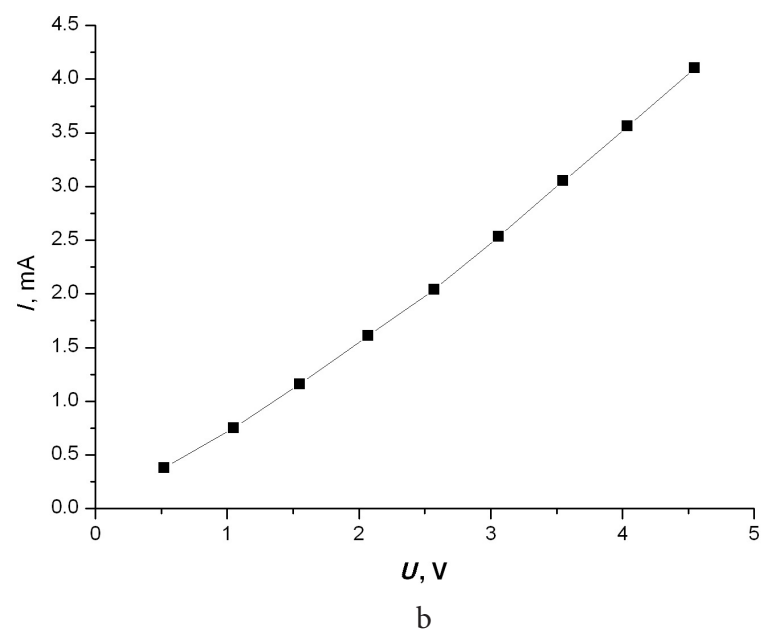

Рис. 4. Вольтамперные характеристики образцов 88\%LSMO/12\%LDPE (a), и 85\%LSMO/14\%LDPE (b).

Fig. 4. Volt-ampere characteristics of samples $88 \%$ LSMO/12\%LDPE (a), and 85\%LSMO/14\%LDPE (b). 


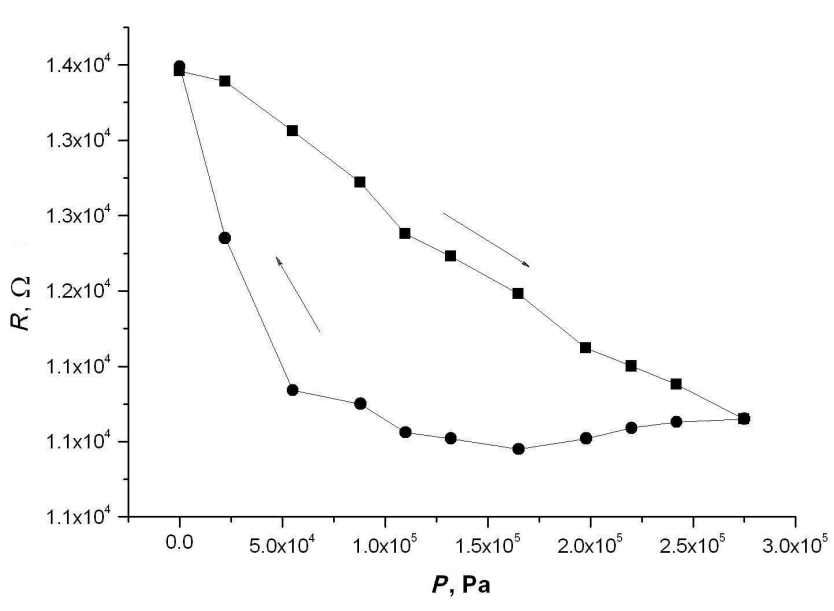

Рис. 6. Зависимость электрического сопротивления образца 85\%LSMO/15\%LDPE от приложенного давления. Стрелками отмечен прямой и обратный ход.

Fig. 6. The dependence of the electrical resistance of the sample $85 \% \mathrm{LSMO} / 15 \% \mathrm{LDPE}$ from applied pressure. The arrows indicate the direct and reverse course.

в связи с наличием релаксационных процессов, характерных для полиэтилена, составляло 100 секунд [9]. Уменьшение электрического сопротивления образцов достигает 17\% при увеличении давления на 2.7 $10^{5}$ Па. В исследованной области давлений чувствительность образца 85\%LSMO/15\%LDPE достигает $8.40 \mathrm{M} \Omega /$ Па.

Исследование отрицательной изотропной магниторезистивности $(M R)$ приготовленных композитов LSMO/LDPE проведено в постоянном магнитном поле, напряженностью до 15 кЭ. Значения $M R$ в исследованных нами образцах меньше, чем величины $M R$ в керамических составах LSMO/ $\mathrm{Sb}_{2} \mathrm{O}_{3}$, синтезированных по одношаговой технологии [3], но сравнимы с результатами работ по изучению магниторезистивности в полимерных композитах на основе LSMO [5]. Подобные материалы отличаются простотой приготовления, в отличие от многослойных магнитных сенсоров на основе магнитоэлектрического эффекта [18]. Наибольшие величины магниторезистивности показали составы $90 \% \mathrm{LSMO} / 10 \% \mathrm{LDPE}$ и $88 \% \mathrm{LSMO} / 12 \% \mathrm{LDPE}$. Для них магниторезистивность от напряженности магнитного поля зависит линейно и достигает $2 \%$ при 15 кЭ.

Таким образом, синтезированные полимерные композиты, изменяющие свое электрическое сопротивление, как под действием одноосного давления, так и во внешних постоянных магнитных полях, могут являться основой для многофункциональных сенсоров давления и постоянного магнитного поля.
Благодарности/Acknowledgements. Авторь признательны профессорам Гавриляченко В.Г., Торгашеву В. И., Тополову В. Ю. за иенные замечания./Authors are grateful to professors Gavrilyatchenko V. G., Torgashev V.I., Topolov V. Yu. for valuable remarks.

\section{Литература/References}

1. H.Y. Hwang, S.-W. Cheong, N.P. Ong, B. Batlogg. Phys. Rev. Lett. 77 (10), 2041 (1996). Crossref

2. Ll. Balcells, A.E. Carrillo, B. Martinez, J. Fontcuberta. Appl. Phys. Lett. 74 (26), 4014 (1999). Crossref

3. Yu. V. Kabirov, V.G. Gavrilyathenko, A.S. Bogatin, T.I. Chupachina, T.V. Gavrilyatchenko. Physics of the Solid State. 57 (1), 16 (2015). (in Russian) [Ю.В.Кабиров, В.Г. Гавриляченко, А.С. Богатин, Т.И. Чупахина, Т. В. Гавриляченко. ФТТ. 57 (1), 16 (2015).]

4. C. Artale, S. Fermepin, M. Forti, M. Latino, M. Quintero, L. Granja, J. Sacanell, G. Polla, P. Levy. Physica B. 404, 2760 (2009). Crossref

5. L. Yang, X. Yang, C. Cheng, L. Lv, Y. Zhao. Physics Procedia. 27, 92 (2012). Crossref

6. X. Zhang, Z. Yao, Z. Ge, K. Yao, R. Tao, T. Yu, J. Han. Journal of Testing and Evaluation. 45 (1), 303 (2017). $\underline{\text { Crossref }}$

7. S. Majumdar, H. S. Majumdar, R. Laiho and R. Osterbacka. New Journal of Physics. 11, 013022 (2009). Crossref

8. M.H.G. Wichmann, S.T. Buschhorn, J. Gehrmann, K. Schulte. Phys. Rev. B. 80, 245437 (2009). Crossref

9. X.-W. Zhang, Y. Pan, Q. Zheng, X.-S. Yi. J. Polym. Sci. Part B. Polymer Physics.38, 2739 (2000). Crossref

10. A. Tager. Fiziko-khimiya polimerov. Moscow, Scientific World (2007) 573 p. (in Russian) [А. А. Тагер. Физикохимия полимеров. Москва, Научный мир (2007) 573 с.]

11. E.A. Filonova, A.N. Demina, E.A. Kleibaum, L.Y. Gavrilova, A.N. Petrov. Inorganic Materials (translated from Neorganicheskie Materialy). 42, 443 (2006). Crossref

12. P. Aldebert, J. P. Traverse. Materials Research Bulletin. 14, 303 (1979). Crossref

13. S. Geller. Acta Cryst. B27, 821 (1971). Crossref

14. Z. Yuheng, L. Xijun, L. Kebin, Z. Kaigui, Z. Jingsheng. J. Appl. Phys. 81, 6943 (2002). Crossref

15. S. Krimm, C. Y. Liang, G. B. B. M. Sutherland. The journal of chemical physics. 25, 549 (1956). Crossref

16. S. Kirkpatrick. Rev. Mod. Phys. 45 (4), 574 (1973). Crossref

17. C. Hofener, J.B. Philipp, J. Klein, L. Alff, A. Marx, B. Buchner, R. Gross. Europhys. Lett. 50 (5), 681 (2000). Crossref

18. M.I. Bichurin, V. M. Petrov, R. V. Petrov, Yu. V. Kiliba, F. I. Bukashev, A.Y. U. Smirnov, D.N. Eliseev. Ferroelectrics. 280, 199 (2002). Crossref 